\title{
Vitamin C Determination by Ultraviolet Spectroscopy and Multiproduct Calibration
}

\author{
Dayane A. Santos, Karen P. Lima, Paulo H. Março and Patrícia Valderrama* \\ Universidade Tecnológica Federal do Paraná (UTFPR), CP 271, 87301-899 Campo Mourão-PR, Brazil
}

\begin{abstract}
In this work the vitamin $\mathrm{C}$ was determined in industrialized nectar juices through ultraviolet (UV) spectroscopy and multiproduct multivariate calibration, based on partial least squares (PLS) regression. Since samples with different flavors, sugar content (light or not) were together in the model construction, it can be considered as a multiproduct and, due to the heterogeneity of the samples, it was necessary to optimize the calibration and validation sets by outliers elimination. The model was developed and validated by the evaluation of the figures of merit such as: accuracy, sensitivity, analytical sensitivity, adjust, linearity, relative prediction deviation, limits of detection and quantification, indicating that the multiproduct model developed from UV spectroscopy and PLS regression can be used in the industrial routine analysis as an alternative to titration or other time and reagent consuming methods. Here, it was evidenced that the UV-PLS multiproduct model provides advantages as being free of sample preparation steps, is suitable to be updated in order to measure other parameters, does not generates residues and is feasible to be implemented for on-line monitoring. Furthermore, the application of multivariate calibration in multiproduct models is extremely attractive from the industrial point of view.
\end{abstract}

Keywords: vitamin C, UV spectroscopy, multiproduct calibration, PLS

\section{Introduction}

Vitamin C or L-ascorbic acid is an essential nutrient for human health, ${ }^{1}$ widely known for its potent antioxidant properties. It can be used in high-doses and has being pointed out as presenting benefits in the treatment of Alzheimer's disease. ${ }^{2}$ Among the multiple roles played by this vitamin, its primary functions are to act as a cofactor for reactions requiring reduced iron or copper metalloenzyme and as a protective antioxidant which better reacts in aqueous phases, both intra- and extracellularly. Scientific evidences show that ascorbic acid plays an important rule on the corneal epithelium, the stroma and the endothelium, acting over the maintenance of its functions and ultra-structures. ${ }^{3}$ Furthermore, epidemiologic studies have associated greater plasma vitamin C levels with reduced risk of chronic diseases, including cancer and cardiovascular disease, as well as higher physical performance in the elderly. ${ }^{4}$

The wide variety of ready-to-drink beverages has driven market to give special attention to it, ${ }^{5}$ and so, fruit nectar, which is by definition a non-fermented ready-to-drink beverage, obtained from the edible part of the fresh fruit

*e-mail: patriciav@utfpr.edu.br diluted in water, in which may or not be added of sugars and acids. The preference for beverages classified as nectars has trend to grow due to its convenience and low price and, besides the wide range of flavors commercially available, has the possibility for being used in the production of lowcalorie beverages (classified as light ones) in a feasible way to the industries. These low-calorie beverages are referred to the ones in which the sugars content, normally added in conventional beverages, are entirely replaced by natural or artificial sweeteners. ${ }^{6}$ In both cases, conventional or low-calorie fruit nectars, vitamin $\mathrm{C}$ is used as a conserving reagent.

Nowadays, several methods based on high performance liquid chromatography (HPLC) have already being proposed in order to determine vitamin $\mathrm{C}$ content in beverages, ${ }^{7,8}$ and foods. ${ }^{9}{ }^{10}$ Nonetheless, methods such as capillary zone electrophoresis, ${ }^{11}$ and voltammetry have being used to determine vitamin $\mathrm{C}$ in different samples, as pharmaceutical preparations and fresh fruit juice. ${ }^{12}$ Recently, ultra performance liquid chromatography (UPLC) and HPLC were proposed to determine vitamin $\mathrm{C}$ in beverages and vitamin tablets. ${ }^{13}$

Ultraviolet (UV) spectroscopy can be used together with multivariate calibration to determine various compounds, since the spectral region used could bring information 
about the chemical structures of the compounds due to chromophore absorptions. ${ }^{14}$ By ensuring this, the spectral region can be analyzed with the aid of chemometric methods of multivariate calibration to quantify different constituents in samples such as beverages. ${ }^{14,15}$ Nonetheless, the Association of Official Analytical Chemists, ${ }^{16}$ proposes that, when vitamin $\mathrm{C}$ is found in low concentration, as in fruit juices, the Tillman's method is recommended. This method is based on the reduction of the 2,6-dichlorophenol indophenol by the vitamin $\mathrm{C}$ and was used in this research as reference method, which results were used for multiproduct multivariate calibration (i.e., only one multivariate calibration model to predict the same property of interest in different products) to UV spectroscopy in order to determine vitamin $\mathrm{C}$ content in industrialized nectar juices.

From these observations, the aim of the present study was to evaluate a multiproduct multivariate calibration model derived from UV spectroscopy to determine vitamin $\mathrm{C}$ in beverages from different fruit nectar. In this purpose, the model is a multiproduct because samples with different flavors and differing on sugar content (conventional and light) were together in the model construction.

The local calibration models of one-product have some disadvantages as a large number of computations required for each prediction in routine analysis, besides the fact that each specific calibration equation can only be used for a small population of samples and each sample must be clearly identified to be able to select the best prediction equation. ${ }^{14,17}$ In addition, maintenance of the one-product multivariate calibration models can be as laborious as the multi-product ones. Thus, to save time on updates, it is worthwhile to investigate if multi-product models can be developed..$^{14,18}$

Multiproduct multivariate calibration can be used to construct a multivariate calibration model from different products. In our case, juices of different flavors were used to provide a particular parameter, the vitamin C content. The early studies came from nineties decade, ${ }^{19,20}$ and the goals were evaluations of the performance of new algorithms. Posteriorly, in 2000 and 2006, ${ }^{17,18}$ the multiproduct calibration was recovery but using non-linear calibration methods, which are not easily implemented in practical applications, such as routine analysis in industry. Recently, preliminary studies combining UV spectroscopy and linear method based on partial least squares (PLS) to construct multiproduct multivariate calibration model was proposed..$^{14}$ In those study, vitamin $\mathrm{C}$ and carbohydrates were determined in industrialized juices, but only yellow samples were employed to build the model. In another study the total acidity was evaluated in nectar juice, suggesting the possibility to build multiproduct calibration models by the PLS regression method. ${ }^{6}$

\section{Experimental}

\section{Samples and reagents}

A total of 123 samples of fruit nectar were purchased in different Brazilian marketplaces. The flavors employed were: grape ( 9 samples), white grape (3 samples), light grape (6 samples), peach (9 samples), light peach (6 samples), pear (3 samples), strawberry (3 samples), passion fruit (9 samples), light passion fruit ( 3 samples), mango (9 samples), light mango (6 samples), apple (12 samples), lemon (3 samples), orange (12 samples), light orange (3 samples), guava (6 samples), light guava (6 samples), cashew (3 samples), light cashew (6 samples) and pineapple (6 samples). Table S1, in Supplementary Information, shows the sample compositions.

The following reagents were used for the Tillman's method: ascorbic acid (Impex, Wood Dale, USA), 2,6-dichlorophenol indophenol sodium salt (SigmaAldrich, St. Louis, USA), indigo carmine (Sigma-Aldrich, St. Louis, USA), metaphosphoric acid, glacial acetic acid and hydrochloric acid (Vetec, Rio de Janeiro, Brazil), sodium bicarbonate, sodium hydroxide and potassium biphthalate (Alphatec, Macaé, Brazil), phenolphthalein 1\% (Sigma-Aldrich, St. Louis, USA).

\section{Tillman's method}

Initially, 3 solutions were prepared as follows: solution 1: acid solution, prepared by solubilizing $15 \mathrm{~g}$ of metaphosphoric acid in $40 \mathrm{~mL}$ of glacial acetic acid and then adding $450 \mathrm{~mL}$ of distilled water, being stirred and filtered; solution 2: vitamin $\mathrm{C}$ solution, prepared by solubilizing $100 \mathrm{mg}$ of vitamin $\mathrm{C}$, previously dried, in $100 \mathrm{~mL}$ of the solution 1 in a volumetric flask $(100 \mathrm{~mL})$ and then, diluted 10 times in the same acid solution; solution 3: Tillman's solution, prepared by solubilizing $42 \mathrm{mg}$ of sodium bicarbonate in $50 \mathrm{~mL}$ of distilled water, adding $50 \mathrm{mg}$ of 2,6-dichlorophenol indophenol sodium salt was under stirring until total dissolution of the dye. Then, this solution was filtered and diluted in $200 \mathrm{~mL}$ of distilled water in a volumetric flask.

In the standardization of Tillman's solution, $4 \mathrm{~mL}$ of the solution 2 was used together with $6 \mathrm{~mL}$ of the solution 1 in an Erlenmeyer flask, where $50 \mathrm{~mL}$ of distilled water was added. This solution was titrated with Tillman's solution (solution 3). A blank test was performed, replacing vitamin $\mathrm{C}$ solution (solution 2) by acid solution 
(solution 1) in order to calculate the Tillman's factor (F) according to equation 1 :

$\mathrm{F}=\frac{\text { Mass of vitamin } \mathrm{C} \text { used in the tritation }(\mathrm{mg})}{\text { Volume of Tillman's solution used in tritation }(\mathrm{mL})}$

The titration of the fruit nectar was done by using $40 \mathrm{~mL}$ of filtered sample mixed with $40 \mathrm{~mL}$ of the solution 1 . From this mixture it was taken $10 \mathrm{~mL}$, which was then titrated with the Tillman's solution. The vitamin $\mathrm{C}$ content was calculated through the equation 2 :

Vitamin $\mathrm{C}$ content $(\mathrm{mg}$ per $100 \mathrm{~mL})=\frac{\mathrm{V} \times \mathrm{F} \times 100}{\mathrm{~A}}$

where, $\mathrm{V}$ is the Tillman's solution volume $(\mathrm{mL})$ used in the titration, $\mathrm{F}$ is the Tillman's factor and $\mathrm{A}$ is the sample volume $(\mathrm{mL})$.

Tests were carried out to detect interferent ions as $\mathrm{Fe}^{2+}$, $\mathrm{Sn}^{2+}$ and $\mathrm{Cu}^{2+}$. To this, two drops of methylene blue $0.05 \%$ were added in $10 \mathrm{~mL}$ of a solution $1: 1(\mathrm{v} / \mathrm{v})$, containing fruit nectar and solution 1.

\section{Apparatus, software and multiproduct multivariate calibration}

The spectra were obtained with an Ocean Optics spectrophotometer model USB-650 UV-VIS (Dunedin, USA) and using a $1 \mathrm{~mm}$ quartz cuvette. All spectra were obtained in the range from 200 to $400 \mathrm{~nm}$ (1 nm step) without any sample preparation. The spectra were obtained once the sample containers were opened, and then there was no storage. The data were treated in MATLAB version R2007b (The Math-Works, Natick, USA). The PLS calculations were carried out with the PLS-Toolbox version 5.2. The outliers detection and figures of merit calculations were carried out with a homemade program developed in the laboratory.

The PLS method has been discussed in detail in relevant references. ${ }^{21-23} \mathrm{In}$ this case, the data matrix $\mathrm{X}$ was constituted by the UV spectra of nectar fruit samples and the vector $y$ contained the reference values for vitamin C content, obtained from the Tillman's method. The model was developed with mean center pre-processing.

Sometimes, samples behaving different from the bulk of the data (outliers) can occur by different reasons, such as laboratory error, samples from another population, instrument error and others. ${ }^{22}$ In this research the outliers were detect based on leverage, ${ }^{24}$ unmodeled residuals in spectra, ${ }^{22,24}$ and unmodeled residuals in dependent variables. ${ }^{22}$ The leverage and unmodeled residuals in spectra and dependent variable were evaluated at the calibration set, while the outliers were evaluated by the leverage and unmodeled residuals in spectra at the validation set, as suggested by American Society for Testing and Materials (ASTM E1655-05). ${ }^{24}$

It is not uncommon, when outliers are eliminated in a first model and the model is rebuilt, to find new outliers in this second model. ${ }^{25}$ In this research, it was also verified and the outliers test was relaxed: (i) the first model was built on an initial calibration set; (ii) outliers were detected based on leverage, unmodeled residuals in spectra and dependent variables were removed; (iii) a second model was built, with the same variables latent number; (iv) outliers detected based on leverage, unmodeled residuals in spectra and dependent variables were removed from the second model; (v) a third model was built, with the same variables latent number; (vi) outliers in the validation set were evaluated.

The validation of the proposed multiproduct multivariate model was certified through the determination of figures of merit, such as, accuracy, linearity, sensitivity, analytical sensitivity, adjustment, limits of detection and quantification. The determination was done based on previous publications, ${ }^{25,26}$ and the equations were presented in the Table 1.

The residual prediction deviation (RPD) was utilized to estimate the predictive ability of the model. ${ }^{27}$ This parameter is more suitable for absolute comparisons and can be estimated for calibration $\left(\mathrm{RPD}_{\text {cal }}\right)$ and validation $\left(\mathrm{RPD}_{\text {val }}\right)$ as shown in Table 1.

\section{Results and Discussion}

The calibration and validation data sets were composed by 94 and 29 samples, respectively, selected by the Kennard-Stone algorithm. ${ }^{28}$ According to this algorithm, the first sample selected is the one with largest distance from the center of the data, and the next sample is the most far from the first sample, and so on, until completing the selected number of samples for the calibration set. In this way, Kennard-Stone algorithm was used within each group of samples to ensure that all flavors, light and conventional juices were well represented in calibration and validation sets. The Figure 1 shows the spectra of all samples in the spectral range used in the model development.

The calibration and validation sets were optimized by outliers elimination. In the calibration set, the outliers were eliminated based on data with extreme leverage in calibration, unmodelled residuals in spectral data and unmodelled residuals in property of interest, which in this case was the vitamin $\mathrm{C}$. The outliers in validation set were eliminated based on extreme leverage and unmodelled 
Table 1. Equations 3 to 11 for figures of merit

\begin{tabular}{|c|c|c|}
\hline Figure of merit & Equation & \\
\hline \multirow{2}{*}{ Accuracy } & $\operatorname{RMSEP}^{\mathrm{a}}=\sqrt{\frac{\sum_{\mathrm{i}=1}^{\mathrm{nv}}\left(\mathrm{y}_{\mathrm{i}}-\hat{\mathrm{y}}_{\mathrm{i}}\right)^{2}}{\mathrm{nv}}}$ & (3) \\
\hline & $\mathrm{RMSEC}^{\mathrm{b}}=\sqrt{\frac{\sum_{\mathrm{i}=1}^{\mathrm{nc}}\left(\mathrm{y}_{\mathrm{i}}-\hat{\mathrm{y}}_{\mathrm{i}}\right)^{2}}{\mathrm{nc}-\mathrm{nVL}+1}}$ & (4) \\
\hline \multirow{2}{*}{ Relative prediction deviation } & $\mathrm{RPD}_{\text {cal }}^{\mathrm{c}}=\frac{\mathrm{DP}_{\text {cal }}}{\mathrm{RMSECV}}$ & (5) \\
\hline & $\mathrm{RPD}_{\text {val }}^{\mathrm{d}}=\frac{\mathrm{DP}_{\mathrm{val}}}{\mathrm{RMSEP}}$ & (6) \\
\hline Sensitivity & sensitivity $=\frac{1}{\left\|\mathbf{b}^{\mathrm{e}}\right\|}$ & (7) \\
\hline Analytical sensitivity & analytical sensitivity $=\frac{\text { sensitivity }}{\left\|\delta \mathrm{x}^{\mathrm{f}}\right\|}$ & (8) \\
\hline Analytical sensitivity ${ }^{-1}$ & analytical sensitivity $^{-1}=\frac{1}{\text { analytical sensitivity }}$ & $(9)$ \\
\hline Limit of detection & limit of detection $=3.3 \delta \mathrm{x}\|\mathbf{b}\|=3.3 \delta \mathrm{x} \frac{1}{\text { sensitivity }}$ & (10) \\
\hline Limit of quantification & limit of quantification $=10 \delta \mathrm{x}\|\mathbf{b}\|=10 \delta \mathrm{x} \frac{1}{\text { sensitivity }}$ & $(11)$ \\
\hline \multicolumn{3}{|c|}{ 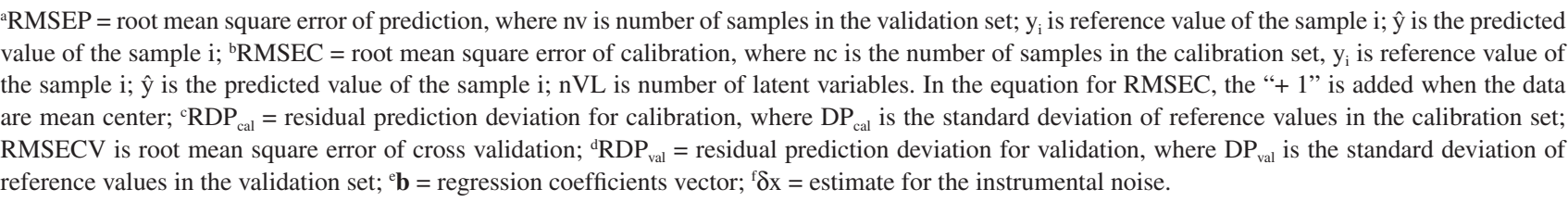 } \\
\hline
\end{tabular}

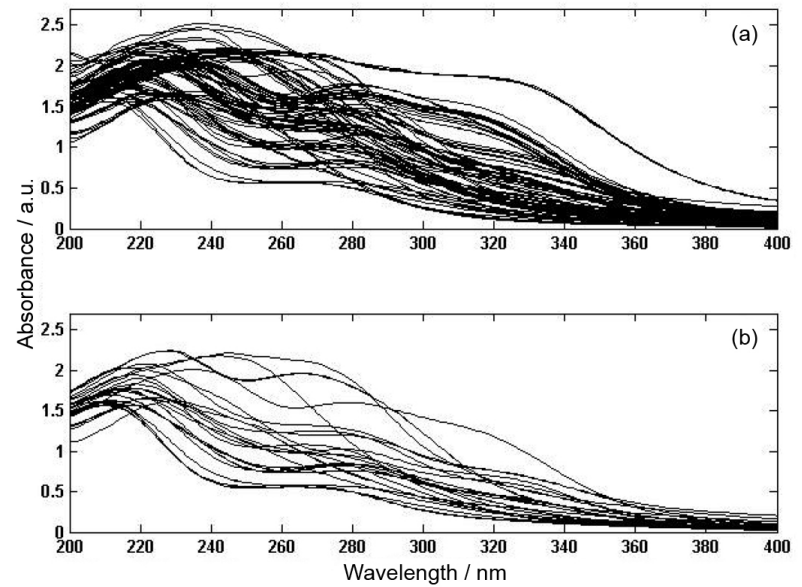

Figure 1. UV spectra of the industrialized nectar juices; (a) calibration data set; and (b) validation data set.

residuals in spectral data. This procedure resulted in 79 and 25 samples for calibration and validation, respectively.

Root mean square error of cross validation (RMSECV) was utilized to select the optimum model dimension. In this case, the minimum RMSECV for the calibration samples, obtained by contiguous block cross validation of nine samples, result the choice of four latent variables for mean-centered model development. The figures of merit for the model are shown in Table 2.

Table 2. Analytical figures of merit for the multiproduct PLS model

\begin{tabular}{lcc}
\hline Figure of merit & & UV model \\
\hline & RMSEC $^{\mathrm{c}}$ & 4.2504 \\
Accuracy $^{\mathrm{a}}$ & RMSEP $^{\mathrm{d}}$ & 3.0945 \\
& RMSECV $^{\mathrm{e}}$ & 4.4049 \\
\hline Relative prediction deviation $^{\mathrm{b}}$ & $\mathrm{RPD}_{\text {cal }}^{\mathrm{f}}$ & 2.8589 \\
\hline Sensitivity $^{\mathrm{a}}$ & $\mathrm{RPD}_{\text {val }}^{\mathrm{g}}$ & 2.4220 \\
Analytical sensitivity $^{-1} \mathrm{a}$ & & 0.1110 \\
Fit correlation coefficient & & 0.3315 \\
Limit of detection $^{\mathrm{a}}$ & & 0.8890 \\
Limit of quantification $^{\mathrm{a}}$ & & 1.0941 \\
\hline
\end{tabular}

${ }^{\mathrm{a}}$ Results in mg per $100 \mathrm{~mL}^{-1}$; ${ }^{\text {bdimensionless units; }{ }^{\mathrm{C}} \mathrm{RMSEC}=\text { root mean }}$ square error of calibration; ${ }^{\mathrm{R} M S E P}$ : root mean square error of prediction; ${ }^{\mathrm{e}} \mathrm{RMSECV}=$ root mean square error of cross validation; ${ }^{\mathrm{r}} \mathrm{RDP}_{\text {cal }}=$ residual prediction deviation for calibration; ${ }^{\mathrm{g} D P} \mathrm{val}_{\mathrm{val}}=$ residual prediction deviation for validation. 
Accuracy represented by root mean square error of calibration (RMSEC), root mean square error of prediction (RMSEP) and RMSECV showed that model dimension was properly chosen and the model was not over fitted. These parameters incorporate random and bias errors. Then, accuracy can also be represented by the fit of the reference values against the predicted ones and the slope, the intercept, correlation coefficient. ${ }^{26}$ Figure 2 shows the fit of the multiproduct model, presented by plotting the reference values against the estimated values for vitamin $\mathrm{C}$. The slope and intercept for this linear fit are also presented in the Figure 2, while the correlation coefficient is presented in Table 2. The correlation coefficient value, 0.8890 , was considered satisfactory since previous research reported coefficient value around 0.7 when the reference method is the titration method. ${ }^{6,25,29}$

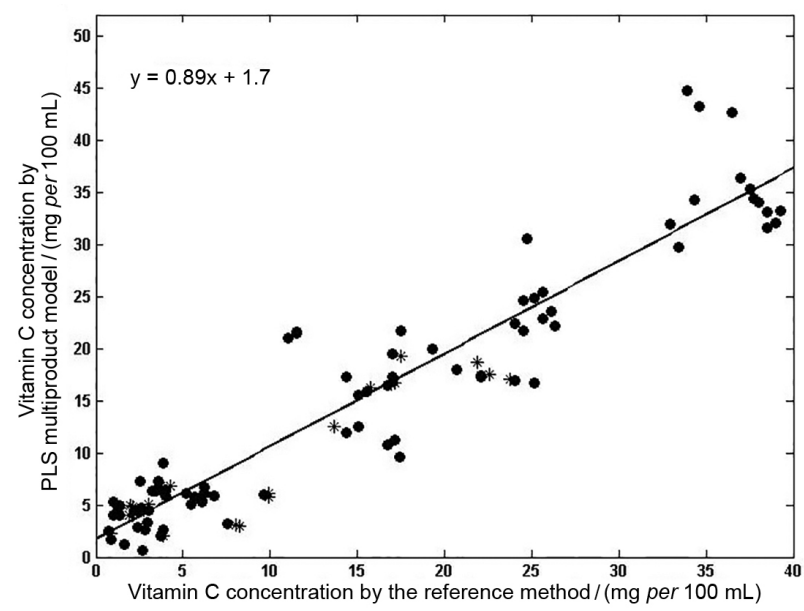

Figure 2. Reference values against the values estimated by the PLS multiproduct model. Calibration samples $(\bullet)$; validation samples $(*)$.

Residual prediction deviation (RPD) is the ratio of natural variation in the samples to the size of probable errors occurring during the prediction, and it is more useful for comparing models on different data sets or in absolute terms. It was calculated for the calibration and validation sets and presented values of 2.86 and 2.42 for calibration and prediction, respectively, which was above 2.4 , the lower limit desired for calibration equations. ${ }^{30}$

The sensitivity, presented in Table 1, showed appropriate results taking into account the analytical range of the model (0.74-39.25 mg per $100 \mathrm{~mL}$ ). However, because of the preprocessing used in PLS model development, the analytical sensitivity is more suitable for evaluate the sensitivity of an multivariate calibration method. Considering a perfect fit of the model and that the spectral noise represent the large source of error, the inverse of the analytical sensitivity (or analytical sensitivity ${ }^{-1}$ ) allows for the establishment of a minimum concentration difference which is discernible by the analytical method in the range of concentrations where it was applied. ${ }^{27}$ Based on this, it is possible to distinguish samples with concentration difference of vitamin C of $0.33 \mathrm{mg}$ per $100 \mathrm{~mL}$.

Residuals plot from calibration and validation samples are shown in Figure 3, and were used to evaluate linearity of the multiproduct model. The absolute and relative errors (most of samples around 20\%) are randomly distributed. These results suggest that this data set fit on a linear model. ${ }^{22,27}$ To confirm the model linearity, Figure 4 show the histogram for the student residuals whose distribution resemble a Gaussian behavior. The Jarque-Bera test confirm that the student residuals are normally distributed at $95 \%$ of confidence level. ${ }^{24,31}$
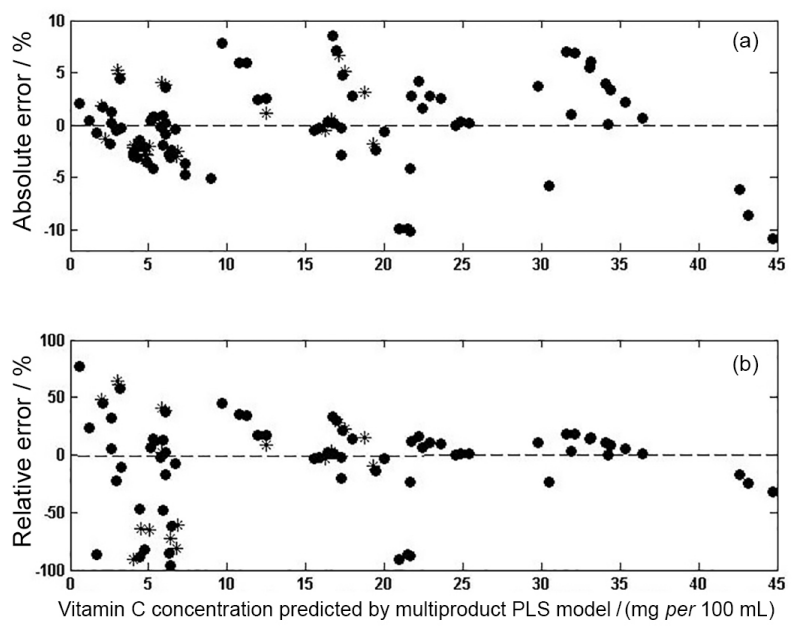

Figure 3. Residuals plot for the multiproduct multivariate PLS model; (a) in terms of the absolute errors; (b) in terms of relative errors. Calibration samples $(\bullet)$; validation samples $(*)$.

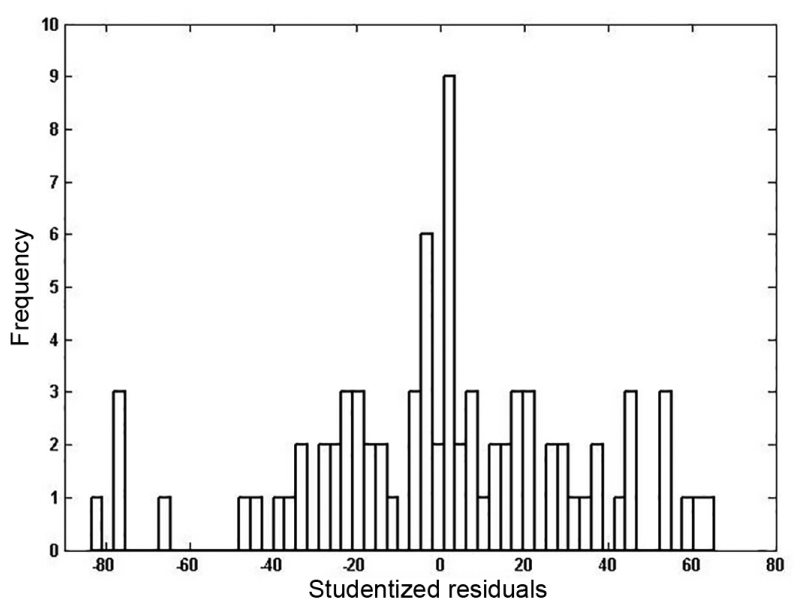

Figure 4. Histogram of the student residuals.

Limits of detection and quantification for the multiproduct model show results coherent with the measured quantities and the RMSEP obtained. The results obtained for the figures of merit showed that the proposed 
method based on UV spectroscopy and multiproduct multivariate calibration is promising. Furthermore, the UV-PLS is a low cost, fast and sample preparation free methodology.

\section{Conclusions}

A method to determine vitamin C by UV spectroscopy and multiproduct multivariate calibration based on PLS is suitable to be constructed. The multiproduct model was optimized by outlier detection and it was validated by calculation of the figures of merit, showing promising results. The model showed an appropriate sensitivity capacity and values for accuracy, limits of detection and quantification, besides other figures of merit presented results which indicates that the multiproduct model developed by UV spectroscopy can be used in the industrial routine analysis as an alternative to titration or even others methods. By comparing with the traditional ones, the UV-PLS multiproduct model present advantages as no sample preparation, capacity for being updated to others parameters, is waste free and present possibilities for online monitoring.

\section{Supplementary Information}

Supplementary data are available free of charge at http://jbcs.sbq.org.br as PDF file.

\section{Acknowledgments}

The authors thank CAPES (Coordenação de Aperfeiçoamento de Pessoal de Nível Superior) for fellowship to Dayane A. Santos.

\section{References}

1. Sapei, L.; Hwa, L.; Procedia Chem. 2014, 9, 62.

2. Murakami, K.; Irie, K.; Shimizu, T. In Diet and Nutrition in Dementia and Cognitive Decline; Martin, C. R.; Preedy, V., eds.; Academic Press: Kidlington, 2014, ch. 61.

3. Serra, H. M.; Suárez, M. F.; Espósito, E.; Urrets-Zavalía, J. A. In Handbook of Nutrition, Diet and the Eye; Preedy V., ed.; Academic Press: Kidlington, 2014, ch. 31.

4. Ishigami, A.; Yokoyama, T. In Aging: Oxidative Stress and Dietary Antioxidants; Preedy, V., ed.; Academic Press: Kidlington, 2014, ch. 12.

5. Zulueta, A.; Esteve, M.; Frasquet, I.; Frígola, A.; Food Chem. 2007, 103, 1365.
6. Santos, D. A.; Lima, K. P.; Março, P. H.; Valderrama, P.; Braz. J. Food Res. 2015, 6, 1.

7. Mattila, P. H.; Hellstrom, J.; McDougall, G.; Dobson, G.; Pihlava, J. M.; Tiirikka, T.; Stewart, D.; Karjalainen, R.; Food Chem. 2011, 127, 1216.

8. Valente, A.; Sanches-Silva, A.; Albuquerque, T. G.; Costa, H. S.; Food Chem. 2014, 154, 71.

9. Spínola, V.; Llorent-Martínez, E. J.; Castilho, P. C.; J. Chromatogr. A 2014, 1369, 2.

10. Mazurek, A.; Jamroz, J.; Food Chem. 2015, 173, 543.

11. Chiari, M.; Nesi, M.; Carrea, G.; Righetti, P. G.; J. Chromatogr. A 1993, 645, 197.

12. Pournaghi-Azar, M. H.; Ojani, R.; Talanta 1997, 44, 297.

13. Klimczak, I.; Gliszczynska-Swiglo, A.; Food Chem. 2015, 175 , 100.

14. Santos, D. A.; Março, P. H.; Valderrama, P.; Braz. J. Anal. Chem. 2013, 12, 495.

15. Martelo-Vidal, M. J.; Vázquez, M.; Food Chem. 2014, 158, 28.

16. Latimer, G. W.; Official Methods of Analysis of the Association of Official Analytical Chemistry, $15^{\text {th }}$ ed.; AOAC: Arlington, 1990, vol. 2, p. 1058.

17. Berzaghi, P.; Shenk, J. S.; Westerhaus, M. O.; J. Near Infrared Spectrosc. 2000, $8,1$.

18. Micklander, E.; Kjeldahl, K.; Egebo, M.; NØrgaard, L.; J. Near Infrared Spectrosc. 2006, 14, 395.

19. Naes, T.; Isaksson, T.; Appl. Spectrosc. 1992, 46, 34.

20. Wang, Z.; Isaksson, T.; Kowalski, B. R.; Anal. Chem. 1994, 66, 249.

21. Geladi, P.; Kowalski, B. R.; Anal. Chim. Acta 1986, 185, 1.

22. Martens, H.; Naes, T.; J. Chemometrics 1989, 4, 441.

23. Brereton, R. G.; Analyst 2000, 125, 2125.

24. ASTM E1655-05: Standards Practices for Infrared, Multivariate, Quantitative Analysis, Pennsylvania, 2005.

25. Valderrama, P.; Braga, J. W. B.; Poppi, R. J.; J. Agric. Food Chem. 2007, 55, 8331.

26. Valderrama, P.; Braga, J. W. B.; Poppi, R. J.; Quim. Nova 2009 , $32,1278$.

27. Botelho, B. G.; Mendes, B. A. P.; Sena, M. M.; Quim. Nova 2013, 36, 1416.

28. Kennard, R. W.; Stone, L. A.; Technometrics 1969, 11, 137.

29. Valderrama, P.; Braga, J. W. B.; Poppi, R. J.; J. Braz. Chem. Soc. 2007, 18, 259.

30. Piantavini, M. S.; Pontes, F. L. D.; Uber, C. P.; Stremel, D. P.; Sena, M. M.; Pontarolo, R.; Spectrochim. Acta, Part A 2014, $125,396$.

31. Jarque, C. M.; Bera, A. K.; Int. Stat. Rev. 1987, 55, 163.

Submitted: November 28, 2015 Published online: March 8, 2016 